\title{
Impact of time of onset of symptom of ST-segment elevation myocardial infarction on 1-year rehospitalization for heart failure and mortality
}

Valeria Paradies MD ${ }^{1}$, Huili Zheng MSc ${ }^{2}$, Mervyn H.H. Chan BSc ${ }^{1,3}$, Mark Y. Chan MBBS, $\mathrm{MHS}^{4}$, David C Foo MBBS ${ }^{5}$, Chee W. Lee MBBS, PhD', Soo T. Lim MBBS ${ }^{1}$, Huay C. Tan MBBS $^{4}$, Jack W. Tan MBBS ${ }^{1}$, Khim L. Tong ${ }^{7}$, Aaron S. Wong MBBS ${ }^{1}$, Philip E. Wong MBBS $^{1}$, Khung K. Yeo MBBS ${ }^{1}$, Ling L. Foo PhD², Terrance S. Chua MBBS ${ }^{1}$, Tian H. Koh MBBS ${ }^{1}$, Heerajnarain Bulluck MBBS, PhD ${ }^{1,8,9 *}$ Derek J Hausenloy MD, PhD ${ }^{1,3,9,10,11,12^{*}}$

*These 2 authors made equal contribution and should be considered as Joint Senior Authors

${ }^{1}$ National Heart Centre Singapore, Singapore, Singapore

${ }^{2}$ National Registry of Disease Office, Health Promotion Board, Singapore

${ }^{3}$ Cardiovascular and Metabolic Disorders Program, Duke-National University of Singapore, Singapore, Singapore

${ }^{4}$ National University Heart Centre, Singapore

${ }^{5}$ Tan Tock Seng Hospital, Singapore, Singapore

${ }^{6}$ Khoo Teck Puat Hospital, Singapore, Singapore

${ }^{7}$ Changi General Hospital, Singapore

${ }^{8}$ Norfolk and Norwich University Hospital, Norwich, UK

${ }^{9}$ The Hatter Cardiovascular Institute, University College London, London, UK

${ }^{10}$ Cardiovascular Research Center, College of Medical and Health Sciences, Asia

University, Taiwan

${ }^{11}$ Yong Loo Lin School of Medicine, National University Singapore, Singapore, Singapore

${ }^{12}$ Tecnologico de Monterrey, Centro de Biotecnologia-FEMSA, Nuevo Leon, Mexico

Competing interests: None

Word count: 2447

Keywords: Circadian, STEMI, heart failure

Funding: Prof Mark Y. Chan receives salary support from a National Medical Research Council Clinician Scientist Award (NMRC/CSA-INV/0001/2016). Prof D J Hausenloy is supported by the Singapore Ministry of Health's National Medical Research Council under its Clinician Scientist-Senior Investigator scheme (NMRC/CSA-SI/0011/2017) and Collaborative Centre Grant scheme (NMRC/CGAug16C006), the Singapore Ministry of Education Academic Research Fund Tier 2 (MOE2016-T2-2-021) and the EU-CARDIOPROTECTION CA16225 Cooperation in Science and Technology (COST) Action

\section{Corresponding author:}

Professor Derek J Hausenloy

Cardiovascular \& Metabolic Diseases Program

Duke-NUS Graduate Medical School Singapore

8 College Road,

Singapore 169857

Singapore

Tel +65 65166719

Email derek.hausenloy@duke-nus.edu.sg 


\section{Highlights}

- Circadian rhythm influences cardiovascular physiology and clinical events.

- STEMI incidence is higher in the morning hours.

- STEMI with evening-night symptoms onset have a 30-50\% higher risk of being admitted for HF within 1 year 


\begin{abstract}
Circadian patterns in ST-segment elevation myocardial infarction (STEMI) patients have been previously reported, but little is known about the impact of time dependence of symptom onset on long-term prognosis. Our study population consisted of 11,731 STEMI patients treated by primary percutaneous coronary intervention (PPCl), enrolled in the Singapore Myocardial Infarction Registry (SMIR). Analysis of STEMI incidence trends over the 24-hour period showed the highest rate of symptom onset in the morning, with the peak incidence at 09:00AM. Patients with symptom onset in between 00:00AM-5:59AM showed the highest prevalence of diabetes $(P=0.010)$ and anterior STEMI $(P<0.001)$ and had the longest ischemic time $(\mathrm{P}<0.001)$. After adjusting for confounders, we found an association between time of symptom onset of STEMI and rehospitalization for heart failure (HF) at 1 year, with symptom onset between 06:00PM-11:59PM and 00:00AM-05:59AM having an estimated $30-50 \%$ higher risk of rehospitalization for HF at 1 year. The results of this study demonstrate for the first time that rehospitalization for HF in STEMI patients treated with PPCI has a dependence on the time of onset of symptoms. This may be an additional risk factor to identify those who warrant closer monitoring and more rigorous optimization of their treatment at follow-up, to improve their outcomes.
\end{abstract}




\section{Introduction}

Circadian rhythm has been shown to influence cardiovascular physiology and clinical events. Heart rate, blood pressure and circulating hormones such as cortisol are well known to follow circadian patterns. ${ }^{1}$ Similarly, several clinical studies have reported time of day dependence for acute myocardial infarction (AMI), ventricular tachycardia, sudden cardiac death and stent thrombosis. ${ }^{2-5}$ The peak incidence of AMI in the early hours of the morning has been attributed to, among others factors, changes in sympathetic tone, coagulation status, platelet aggregability and endothelial function. ${ }^{6}$

Several studies have shown time of day dependence on myocardial infarct (MI) size, by both cardiac enzymes and cardiac magnetic resonance (CMR). ${ }^{7-11}$ Multiple cardiomyocyte clock genes appear to be responsible for the circadian pattern of ischemia reperfusion injury variability and, as a consequence, $\mathrm{MI}$ size. Up to a 3.5 fold variation in mice $\mathrm{MI}$ size has been demonstrated in animal models, depending on the time of coronary occlusion in the 24hour cycle. Acute MI size has been found to be largest in the sleep-to-wake transition. This phenomenon could be suppressed when genetic ablation of the cardiomyocyte circadian clock was applied. ${ }^{12}$

This circadian variation of ischemic tolerance and myocardial injury has also been noted in humans in the setting of ST-segment elevation myocardial infarction (STEMI). ${ }^{7-11}$ Despite conflicting results, the majority of the studies have reported the largest Ml size for patients presenting with symptom onset during the early hours of the morning. However, the influence of time of symptom onset on long-term outcomes following STEMI has not been fully elucidated. The aim of our study was to analyze whether the time of onset of symptoms for STEMI had an impact on 1-year rehospitalization for heart failure (HF) and mortality in a large real-world registry. 


\section{Methods}

The Singapore Myocardial Infarction Registry (SMIR) is a compulsory, nationwide registry of consecutive patients hospitalized for AMI. The SMIR collects epidemiological data on all cases diagnosed in the public and private sector hospitals, with a small number of out-ofhospital AMI deaths certified by medical practitioners in Singapore. ${ }^{13}$ Patients with AMI are identified through hospital discharge diagnoses, claims submission and death records. Detailed individual-level data are extracted from the patients' hospital records by trained registry coordinators.

The data used in our study are from the period 2007-2015 and included all STEMI patients who underwent primary percutaneous coronary intervention (PPCI). STEMI was defined as typical chest pain of at least 20 minutes and significant ST segment elevation $(0.1 \mathrm{mV}$ or 0.2 $\mathrm{mV}$ on 2 adjacent limb or precordial leads, respectively, or new left bundle-branch block) and confirmed subsequently by a rise in cardiac biomarkers.

The time of symptom onset was recorded in the emergency department and then extracted from the clinical notes by the registry coordinators. According to previous studies, time-ofday of STEMI onset was divided into four 6-hour groups: 06:00AM-11:59AM, 12:00PM05:59PM, 06:00PM-11:59PM and 00:00AM-05:59AM. The time intervals were based on the light transition under tropical climate conditions with non-varying day-night cycles in light intensity and accessibility to pre-hospital and hospital care during different times of the day. ${ }^{8-}$ ${ }^{10}$ All patients with unknown time of symptom onset were excluded from our study.

Death information up to 31 December 2016 was obtained from death records from the Death Registry. Rehospitalization information up to 31 December 2014 was obtained from claims submission from the Ministry of Health.

We evaluated all-cause mortality up to 1 year from STEMI onset and rehospitalization for HF up to 1 year from STEMI discharge. The data were adjusted for the confounding variables which may have had an impact on the major endpoints: age, gender, ethnicity, history of diabetes, history of hypertension, smoking status, history of MI or revascularization, anterior 
STEMI, cardiopulmonary resuscitation, Killip class, ischemic time $>180$ minutes and elevated first troponin.

There was no patient lost to follow-up within the period of available data in this compulsory nationwide registry.

All research-related procedures were performed in accordance with the local guidelines and regulations.

The study was approved by the National Healthcare Group Domain Specific Review Board with a waiver of written informed consent granted for research involving analysis of anonymised registry data.

\section{Statistical analysis}

Data were expressed as median for numeric variables and percentages for categorical variables. All comparisons between baseline variables were assessed using Kruskal-Wallis rank test for numeric variables and Chi-square test for categorical variables. Univariable and multivariable cox regression were used to determine the association between time of symptom onset and death. Univariable and multivariable cox regression, adjusted for competing risk from death, were used to determine the association between time of symptom onset and rehospitalization for HF. Given the lowest 1 year mortality and HF rehospitalization rates in the 06:00AM-11:59AM group, all the calculated HRs were obtained comparing this group with the other 3 time intervals during the rest of the day. STEMI incidence, as well as the rate of 1-year mortality and 1-year rehospitalization for HF among them, were plotted by time of symptom onset to visualize the relationship between symptom onset time and events. A smoothed curve, selected from multiple nested poisson regression models of varying complexity based on likelihood ratio test, was superimposed on each plot. For all analyses, $\mathrm{p}<0.05$ was considered statistically significant. All analyses were carried out using STATA (StataCorp. 2013. Stata Statistical Software: Release 13. College Station, TX: StataCorp LP). 


\section{Results}

In total, 12,453 STEMI patients treated by PPCI were enrolled in the SMIR registry between 2007 and 2015. Time of symptom onset was unknown in 722 patients and they were excluded from the analysis.

Baseline characteristics of the remaining 11,731 patients stratified by the time of symptom onset are summarized in Table 1. Notable differences were as follows: patients with symptom onset between 6:00AM-11:59AM were older (median 57.8 years, $\mathrm{P}=0.027$ ) and had higher prevalence of hypertension (53.6\%, $\mathrm{P}=0.001)$; those in the 12:00PM-05:59PM group were more likely to be smokers $(53.1 \%, \mathrm{P}<0.001)$ and to require cardiopulmonary resuscitation $(4.7 \%, \mathrm{P}=0.018)$; those in the $06: 00 \mathrm{PM}-11: 59 \mathrm{PM}$ group were more likely to have Killip class IV (8.7\%, $\mathrm{P}<0.001)$; those in the 00:00AM-05:59AM group were more likely to have diabetes $(29.9 \%, P=0.010)$, presented with anterior STEMI $(52.8 \%, P<0.001)$, had the longest ischemic time (median 206 minutes, $P<0.001$ ) and were more likely to have elevated first troponin $(52.8 \%, \mathrm{P}<0.001)$.

There were 3,787 (32.3\%) patients with symptom onset between 06:00AM-11:59AM, 2,909 (24.8\%) patients with symptom onset between 12:00PM-05:59PM, 2,656 (22.6\%) patients with symptom onset between 06:00PM-11:59PM and 2,379 (20.3\%) patients with symptom onset between 00:00AM-05:59AM. Finer hourly breakdown of STEMI incidence by symptom onset time over the 24-hour period showed the highest rate of symptom onset between 06:00AM-11:59AM, with the peak at 09:00AM (Figure 1).

\section{Impact of time of symptom onset on 1-year outcome}

Graphical trend of 1-year mortality rate over the 24-hour period is shown in Figure 2. 1-year mortality rate was highest in the 00:00AM-05:59AM group (10.9\%), compared to the rate in the 06:00AM-11:59AM (8.8\%), 12:00PM-05:59PM (9.1\%) and 06:00PM-11:59PM (10.3\%) groups $(P=0.026)$ (Table 1). However, the unadjusted risk of death was highest in the 06:00PM-11:59PM group (HR 1.40, 95\% Cl 1.18-1.66) (Table 2) and persisted after adjusting for age, gender, ethnicity, history of diabetes, history of hypertension, smoking 
status, history of $\mathrm{MI}$ or revascularization, anterior STEMI, cardiopulmonary resuscitation, Killip class, ischemic time $>180$ minutes and elevated first troponin (HR 1.30, 95\% CI 1.091.56) (Table 2). This implied that although patients in the 00:00AM-05:59AM group had the highest mortality rate, patients who died in the 06:00PM-11:59PM group survived for the shortest duration. Kaplan-Meier failure curves showing time to death by symptom onset time is displayed in Figure 3.

Graphical trend of 1-year rehospitalization rate for HF over the 24-hour period is shown in Figure 4. Patients in the 00:00AM-05:59AM group experienced the highest rate of 1-year rehospitalization for $\mathrm{HF}(6.6 \%)$, compared to patients in the 06:00AM-11:59AM (3.7\%), 12:00PM-05:59PM (4.7\%) and 06:00PM-11:59PM (5.5\%) groups $(\mathrm{P}<0.001)$ (Table 1). Symptom onset in both the 06:00PM-11:59PM (HR 1.50, 95\% Cl 1.14-1.99) and 00:00AM05:59AM (HR 1.83, 95\% Cl 1.39-2.41) groups were predictors of 1-year rehospitalization for HF (Table 2). After adjusting for age, gender, ethnicity, history of diabetes, history of hypertension, smoking status, history of $\mathrm{MI}$ or revascularization, anterior STEMI, cardiopulmonary resuscitation, Killip class, ischemic time $>180$ minutes and elevated first troponin, symptom onset in both the 06:00PM-11:59PM (HR 1.37, 95\% Cl 1.02-1.84) and 00:00AM-05:59AM (HR 1.51, 95\% Cl 1.14-2.01) groups remained as predictors of 1-year rehospitalization for HF (Table 2).

We further divided the whole cohort into two groups of patients presenting with symptoms lasting longer or shorter than 120 minutes. In the subgroup with shorter ischemic time, symptom onset was no longer a predictor of 1-year mortality and rehospitalization for HF. After adjusting for confounders, symptom onset in the 06:00PM-11:59PM group with ischemic time longer than minutes, remained a predictor of 1 -year mortality (HR $1.40,95 \%$ $\mathrm{Cl}$ 1.14-1.71). In patients with prolonged ischemia, symptom onset remained a predictors of of 1-year rehospitalization for HF in all groups except for 06:00PM-11:59PM group. (Table 3). 
Kaplan-Meier failure curves showing time to rehospitalization for HF by symptom onset time is displayed in Figure 5.

Figure 6 represents the distribution of STEMI by ischemic time in each 6 -hour symptom onset time interval.

\section{Discussion}

To the best of our knowledge, this is the largest study to have investigated the relationship between the time of onset of symptom of STEMI and 1-year outcomes among STEMI patients undergoing PPCl. In our analysis, we confirmed an association between time of symptom onset and rehospitalization for HF at 1 year. After adjusting for confounders, those with symptom onset between 06:00PM-11:59PM and 00:00AM-05:59AM had an estimated $30-50 \%$ higher risk of being admitted for HF within 1 year. However, we did not find a higher 1-year mortality rate in patients with symptom onset between 00:00AM-05:59AM in the adjusted analysis.

In line with previous studies, we reported a higher STEMI incidence in the morning hours. Higher levels of epinephrine and norepinephrine, plasma renin activity and cortisol secretion have been shown in the morning hours. ${ }^{1}$ The enhanced alpha-sympathetic vasoconstrictor activity in the morning may partially explain the increased vascular resistance and blood pressure and contribute to or even trigger the higher onset of acute cardiovascular events in this time window. ${ }^{14}$ Besides circadian pattern of the above-mentioned physiological factors, a temporal association has been shown between augmented platelet aggregability and increased AMI frequency in morning hours. ${ }^{15}$ Previous reports have found a circadian variation in the effectiveness of pharmacological reperfusion with the lowest reperfusion rate in early morning. ${ }^{16}$ In fact, highest levels of plasminogen activator inhibitor-1 as well as decreased fibrinolytic activity have been demonstrated in early morning STEMI presenters. ${ }^{17}$ Previous studies based on both cardiac biomarkers and CMR showed a higher MI size in those patients with symptom during the early hours of the morning. ${ }^{9,11,18,19}$ The identification of clock genes responsible for ischemic reperfusion injury variability has supported these 
findings. ${ }^{12}$ A previous analysis of STEMI patients from the SMIR up to 2009 by Seneviratna et $\mathrm{al}^{19}$ reported higher mean peak $\mathrm{CK}$, acute HF incidence during the index admission, 30day and 1-year mortality rate in patients with symptom onset between 00:00AM-5:59AM. In our analysis based on a larger population in the contemporary era, there were higher prevalence of diabetes and anterior infarction location and longer ischemic time in patients with overnight symptom onset, consistent with larger Ml size. We hypothesized that the higher risk of being admitted for HF within 1 year among patients with symptom onset between 00:00AM-5:59AM was likely due to the larger MI size, while the higher risk of mortality at 1 year among patients with symptom onset between 06:00PM-11:59PM was likely due to the higher presenting Killip class. We further investigated the predictors of mortality and HF at 1 year in the two subgroups of patients presenting with symptoms lasting shorter or longer than 120 minutes. Interestingly, symptom onset remained a predictor of worse prognosis only in the prolonged ischemia subgroup. These findings underline the relevance of the accessibility and quality of prehospital and hospital care during different times of the day in determining STEMI patients' prognosis. We may conclude that the symptom onset out of the regular working hours window may have played a role in the late presentation and therefore in the worse clinical outcomes of patients with overnight symptoms.

\section{Limitations}

Details on PCl procedures and angiographic data were not available as part of this registry. The medications on discharge and changes in medications/ device therapy during the 1-year follow-up were not available. There may have been other confounders that we were not able to adjust for, such as system delays and differences in access to healthcare out of office hours, the so-called "after-hours" effect, whihc might also have had an impact on the 1-year outcomes. 


\section{Conclusions}

In our large, real-world registry, we found an association between time of symptom onset of STEMI and rehospitalization for HF at 1 year, with symptom onset between 06:00PM11:59PM and 00:00AM-5:59AM having an estimated 30-50\% higher risk of being admitted for HF within 1 year. However, this did not impact on 1-year mortality for patients with symptom onset between 00:00AM-05:59AM in the adjusted analysis. Whether patients presenting between 06:00PM-5:59AM should be considered a new risk factor and can be used to identify those who would benefit from closer monitoring and more vigorous optimization of their treatment at follow-up to improve their outcomes, warrants further investigation. 


\section{Author contribution statement}

Valeria Paradies, Huili Zheng, Mervyn H.H. Chan and Heerajnarain Bulluck contributed to conception and design, or acquisition of data, or analysis and interpretation of data.

Mark Y. Chan, David C Foo, Chee W. Lee, Soo T. Lim, Huay C. Tan, Jack W. Tan, Khim L. Tong, Aaron S. Wong, Philip E. Wong, Khung K. Yeo, Ling L. Foo, Terrance S. Chua and Tian $\mathrm{H}$. Koh revised it critically and contributed to implementation of the research.

Prof. Hausenloy was in charge of overall direction and planning. 


\section{References}

1. Panza, J.A., Epstein, S.E. \& Quyyumi, A.A. Circadian variation in vascular tone and its relation to alpha-sympathetic vasoconstrictor activity. The New England journal of medicine 325, 986-990 (1991).

2. Cohen, M.C., Rohtla, K.M., Lavery, C.E., Muller, J.E. \& Mittleman, M.A. Metaanalysis of the morning excess of acute myocardial infarction and sudden cardiac death. The American journal of cardiology 79, 1512-1516 (1997).

3. Mahmoud, K.D., Lennon, R.J., Ting, H.H., Rihal, C.S. \& Holmes, D.R., Jr. Circadian variation in coronary stent thrombosis. JACC. Cardiovascular interventions 4, 183190 (2011).

4. Muller, J.E., Tofler, G.H. \& Stone, P.H. Circadian variation and triggers of onset of acute cardiovascular disease. Circulation 79, 733-743 (1989).

5. Valkama, J.O., Huikuri, H.V., Linnaluoto, M.K. \& Takkunen, J.T. Circadian variation of ventricular tachycardia in patients with coronary arterial disease. International journal of cardiology 34, 173-178 (1992).

6. De Luca, G., et al. Circadian variation in myocardial perfusion and mortality in patients with ST-segment elevation myocardial infarction treated by primary angioplasty. Am Heart J 150, 1185-1189 (2005).

7. Arroyo Ucar, E., Dominguez-Rodriguez, A. \& Abreu-Gonzalez, P. Influence of diurnal variation in the size of acute myocardial infarction. Med Intensiva 36, 11-14 (2012).

8. Fournier, S., et al. Myocardial infarct size and mortality depend on the time of day-a large multicenter study. PloS one 10, e0119157 (2015).

9. Reiter, R., Swingen, C., Moore, L., Henry, T.D. \& Traverse, J.H. Circadian dependence of infarct size and left ventricular function after ST elevation myocardial infarction. Circulation research 110, 105-110 (2012).

10. Suarez-Barrientos, A., et al. Circadian variations of infarct size in acute myocardial infarction. Heart 97, 970-976 (2011).

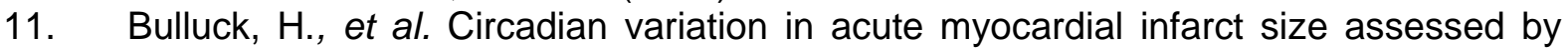
cardiovascular magnetic resonance in reperfused STEMI patients. International journal of cardiology 230, 149-154 (2017).

12. Durgan, D.J., et al. Short communication: ischemia/reperfusion tolerance is time-ofday-dependent: mediation by the cardiomyocyte circadian clock. Circulation research 106, 546-550 (2010).

13. Singapore Myocardial Infarction Registry Report No. 3: Trends In Acute Myocardial Infarction In Singapore 2007 - 2013. (https://www.nrdo.gov.sg/publications/ami 2015).

14. Mores, N., et al. Platelet alpha 2-adrenoceptors and diurnal changes of platelet aggregability in hypertensive patients. J Hypertens 12, 939-945 (1994).

15. Tofler, G.H., et al. Concurrent morning increase in platelet aggregability and the risk of myocardial infarction and sudden cardiac death. The New England journal of medicine 316, 1514-1518 (1987).

16. Kono, T., et al. Circadian variations of onset of acute myocardial infarction and efficacy of thrombolytic therapy. Journal of the American College of Cardiology 27, 774-778 (1996).

17. Fujino, T., et al. Relationship between serum lipoprotein(a) level and thrombin generation to the circadian variation in onset of acute myocardial infarction. Atherosclerosis 155, 171-178 (2001).

18. Mahmoud, K.D., et al. Independent association between symptom onset time and infarct size in patients with ST-elevation myocardial infarction undergoing primary percutaneous coronary intervention. Chronobiol Int 32, 468-477 (2015).

19. Seneviratna, A., et al. Circadian Dependence of Infarct Size and Acute Heart Failure in ST Elevation Myocardial Infarction. PloS one 10, e0128526 (2015). 
